\title{
A Comparative Analysis of Wavelet Families for the Classification of Finger Motions
}

\author{
Jingwei Too ${ }^{1}$, Abdul Rahim Abdullah ${ }^{2}$, Norhashimah Mohd Saad ${ }^{3}$ \\ Fakulti Kejuruteraan Elektrik, Universiti Teknikal Malaysia Melaka, Melaka, Malaysia ${ }^{1,2}$ \\ Fakulti Kejuruteraan Elektronik dan Kejuruteraan Komputer \\ Universiti Teknikal Malaysia Melaka, Melaka, Malaysia ${ }^{3}$
}

\begin{abstract}
Wavelet transform (WT) has been widely used in biomedical, rehabilitation and engineering applications. Due to the natural characteristic of WT, its performance is mostly depending on the selection of mother wavelet function. A proper mother wavelet ensures the optimum performance; however, the selection of mother wavelet is mostly empirical and varies according to dataset. Hence, this paper aims to investigate the best mother wavelet of discrete wavelet transform (DWT) and wavelet packet transform (WPT) in the classification of different finger motions. In this study, twelve mother wavelets are evaluated for both DWT and WPT. The electromyography (EMG) data of 12 finger motions are acquired from online database. Four useful features are extracted from each recorded EMG signal via DWT and WPT transformation. Afterward, support vector machine (SVM) and linear discriminate analysis (LDA) are employed for performance evaluation. Our experimental results demonstrate Bior 3.3 to be the most suitable mother wavelet in DWT. On the other hand, WPT with Bior2.2 overtakes other mother wavelets in the classification of finger motions. The results obtained suggest that Biorthogonal families are more suitable for accurate EMG signals classification.
\end{abstract}

Keywords-Mother wavelet; discrete wavelet transform; wavelet packet transform; electromyography; classification

\section{INTRODUCTION}

Electromyography (EMG) has becoming one of the major interest in the rehabilitation areas due to its usefulness in clinical and human machine interface (HMI) applications [1], [2] Advance in HMI raises the efficiency of control system in myoelectric prosthetic control [3]. By using the surface EMG signals recorded from the skin surface, the myoelectric interface based on EMG pattern recognition allows the amputee and patient to gain control on the artificial hand.

The techniques such as signal processing, feature extraction and classification are usually involving in the EMG pattern recognition. One of the methods that have been widely applied in biomedical signal processing is wavelet transform (WT). From the previous works, WT was found to be the best timefrequency method since it often gave promising results in the classification of EMG signals [4], [5]. Unlike short time Fourier transform (STFT), WT offers flexible time and frequency resolution, which leads to high quality signal information. However, the performance of WT differs from the selection of mother wavelet [6]. In one study, Omari et al. [7] classified eight hand motions using discrete wavelet transform (DWT). The authors reported DWT with Symlet 4 achieved the highest accuracy of $95 \%$. For instance, Phinyomark et al. [8] studied the performance of several mother wavelets in DWT. The authors found that DWT with Daubechies 7 ensured better classification result. On one side, Hariharan et al. [9] made a comparative study of different wavelet families for the classification of wrist motions. The results obtained indicated that wavelet packet transform (WPT) with Coiflet and Biorthogonal families offered superior performance. Another study reported that Daubechies 6 to be the optimal mother wavelet of DWT in EMG pattern recognition [10].

According to literature, it can be inferred that different mother wavelets in WT offered different kind of responses in EMG pattern recognition system. Thus, it is believed that the selection of mother wavelet in WT is remaining insufficient and unclear. In addition, most of the wavelet studies made use of smaller number of motions (less than 10 motions) in the process of evaluations. In fact, a greater number of motion types offers multifunctional myoelectric prosthetic control, which is more preferred by the users [11]. Therefore, in this work, the best mother wavelet of DWT and WPT when discriminating many finger motions are investigated.

In this study, the EMG data of 12 different finger motions are collected form online database. Twelve types of mother wavelets in both DWT and WPT are carefully examined. To obtain the hidden and useful information from the EMG signal, several useful features are extracted from the wavelet coefficients. Finally, the extracting features are fed into the classifiers for performance evaluation, and the best mother wavelet for both DWT and WPT are pointed.

\section{MATERIAL AND METHOD}

\section{A. EMG Data}

In this work, the fourth version of Non-Invasive Adaptive Prosthetic (NinaPro) project is employed [12]. NinaPro project is a publicity access EMG database, which records a huge number of EMG data from multiple subjects. The fourth version of NinaPro database (DB4) comprises of the surface EMG signals recorded from 10 healthy subjects. In DB4, twelve bipolar electrodes were used in the process of recording. Eight electrodes were placed equally around the forearm. Two electrodes were placed on the biceps and triceps brachii muscles. Another two electrodes were placed on the extensor digitorum superficialis and flexor digitorum superficialis muscles. In this study, the surface EMG signals of 12 different finger motions (Exercise A) are utilized. Table I outlines the listed finger motions. In the experiment, each motion was 
performed for 5 seconds, followed by a resting state of 3 seconds. Additionally, each motion was repeated for six times, and the EMG signals were sampled at $2 \mathrm{kHz}$ [12]. Furthermore, all the resting phases are removed.

\section{B. System Overivew}

Fig. 1 demonstrates the flow diagram of proposed EMG pattern recognition system. In the first step, DWT and WPT with 12 different mother wavelets are employed to transform the EMG signals into multiresolution coefficients. Then, four useful features are extracted from each wavelet coefficient to form the feature set. Next, two machine learning algorithms namely support vector machine (SVM) and linear discriminate analysis (LDA) are applied for classification. At the end of the experiment, the best mother wavelet in both DWT and WPT are pointed.

\section{Discrete Wavelet Transform}

In recent days, discrete wavelet transform (DWT) has becomes popular in rehabilitation and clinical areas. Correspondingly, DWT offers good frequency resolution at low frequency components. On the contrary, good time resolution can be obtained at high frequency components [13]. In wavelet decomposition, DWT decomposes the EMG signal into multiresolution coefficients. The decomposition of DWT involves two digital filters, which are low-pass and high-pass filters. The first decomposition of DWT can be defined as:

$$
\begin{aligned}
& D[n]=\sum_{n} x[k] \cdot h[2 n-k] \\
& A[n]=\sum_{n} x[k] \cdot g[2 n-k]
\end{aligned}
$$

where $x[k]$ is the signal, $D[n]$ is the detail and $A[n]$ is referred to approximation. The wavelet decomposition is repeated until the desired level is reached. Within each decomposition, the signal is down-sampled by a factor of 2 [13], [14]. In the past studies, the best DWT wavelet decomposition level to analyze the EMG signals was mostly falling at fourth decomposition level [5], [8], [15]. In this regard, the DWT with fourth decomposition level is used in this work. The sample of DWT with Biorthogonal 3.3 at fourth decomposition level is shown in Fig. 2.

TABLE I. LISTED FINGER MOTIONS

\begin{tabular}{|l|l|}
\hline Label & Finger motion task \\
\hline F1 & Index flexion \\
\hline F2 & Index extension \\
\hline F3 & Middle flexion \\
\hline F4 & Middle extension \\
\hline F5 & Ring flexion \\
\hline F6 & Ring extension \\
\hline F7 & Little finger flexion \\
\hline F8 & Little finger extension \\
\hline F9 & Thumb adduction \\
\hline F10 & Thumb abduction \\
\hline F11 & Thumb flexion \\
\hline F12 & Thumb extension \\
\hline
\end{tabular}

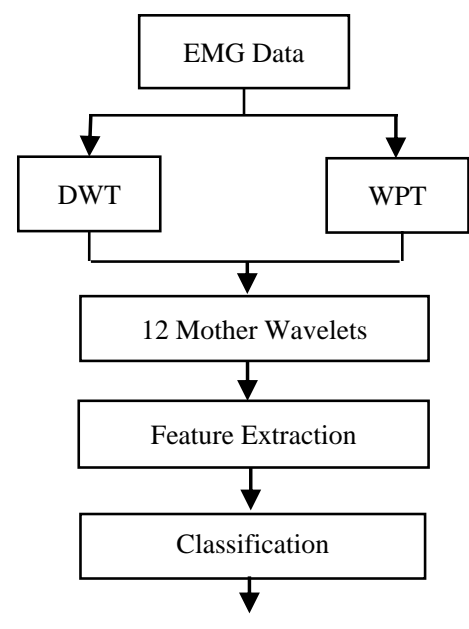

12 different finger motions

Fig. 1. Proposed Recognition System.
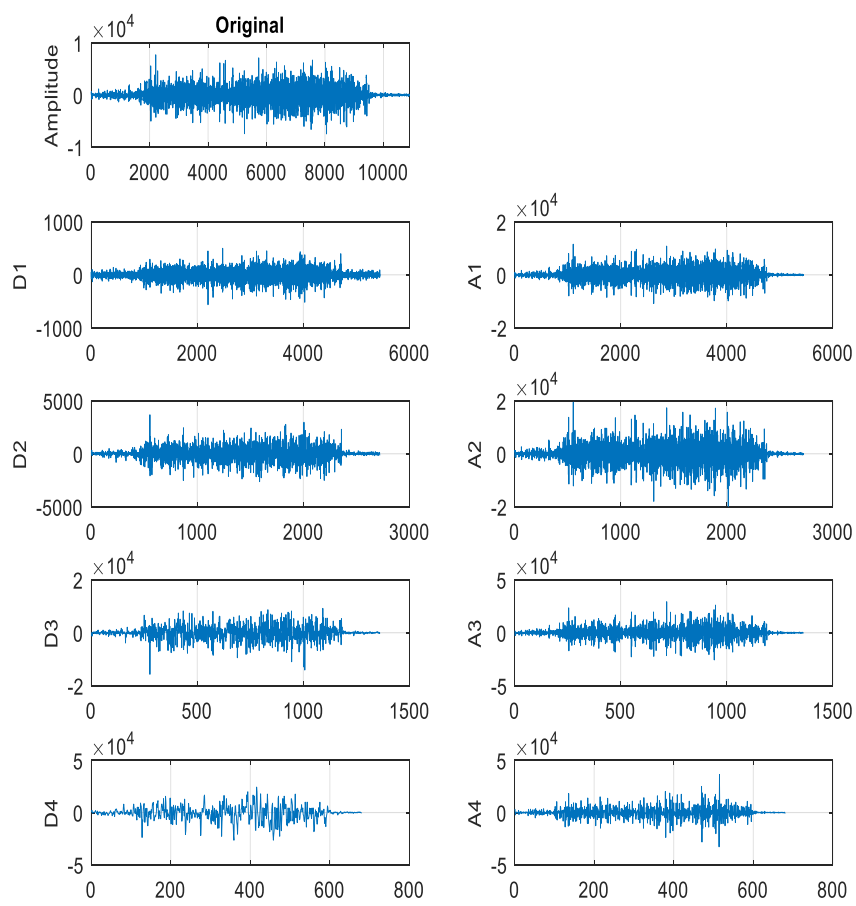

Fig. 2. Wavelet Decomposition of DWT with Biorthogonal 3.3 at Fourth Decomposition Level.

\section{Wavelet Packet Transform}

Wavelet packet transform (WPT) is one of the powerful pre-processing tools in biomedical signal processing [16]. In WPT, higher frequency component has better time resolution, whereas lower frequency component offers better frequency resolution.

Generally, WPT is also known as a tree of sub-spaces that decomposes the signal into two orthogonal bases [17]. Based on previous works, the best decomposition level is found to be 3 [9], [17]. Fig. 3 demonstrates the wavelet decomposition tree at third decomposition level. At each decomposition level, WPT decomposes the signal into two sub-bands involving the 
high and low frequency bands. In decomposition tree, the number of subspaces denotes as $j$ and each of the subspace has its depth $i$. The wavelet packet decomposition of the parent node $(i, j)$ can be expressed as:

$$
\begin{aligned}
& \psi_{i+1}^{2 j+1}(k)=\sum_{n} h[n] \cdot \psi_{i}^{j}\left(k-2^{i} n\right) \\
& \psi_{i+1}^{2 j}(k)=\sum_{n} g[n] \cdot \psi_{i}^{j}\left(k-2^{i} n\right)
\end{aligned}
$$

where $h[n]$ and $g[n]$ are referred to the high pass and low pass filters, respectively. In this work, WPT at third decomposition level is utilized.

\section{E. Mother Wavelet Selection}

Mother wavelet selection is the most critical problem in both DWT and WPT. According to literature, different mother wavelet offers difference performance in different dataset [18], [19]. In fact, a mother wavelet might work properly in dataset $A$, but not to the dataset $B$. In this paper, the performance of 12 different mother wavelets for both DWT and WPT are examined. Table II shows the utilized 12 mother wavelets.

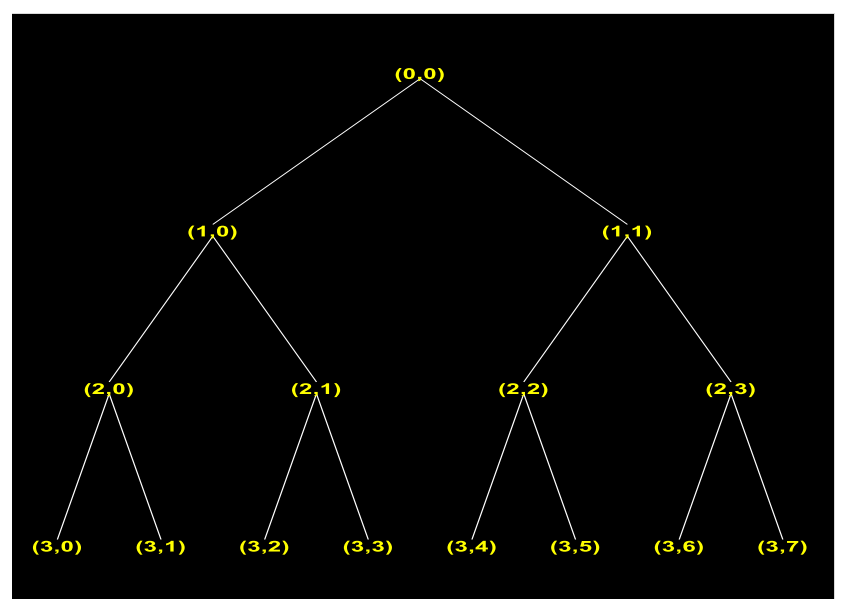

Fig. 3. Wavelet Packet Decomposition Tree at Third Decomposition Level.

TABLE II. TwELVE MOTHER WAVELETS

\begin{tabular}{|l|l|l|l|}
\hline No. & Mother wavelet & No. & Mother wavelet \\
\hline 1 & Biorthogonal 2.2 (Bior2.2) & 7 & Daubechies 4 (Db4) \\
\hline 2 & Biorthogonal 3.3 (Bior3.3) & 8 & Daubechies 6 (Db6) \\
\hline 3 & Biorthogonal 4.4 (Bior4.4) & 9 & Daubechies 8 (Db8) \\
\hline 4 & Coiflet 3 (Coif3) & 10 & Symlet 4 (Sym4) \\
\hline 5 & Coiflet 4 (Coif4) & 11 & Symlet 6 (Sym6) \\
\hline 6 & Coiflet 5 (Coif5) & 12 & Symlet 8 (Sym8) \\
\hline
\end{tabular}

\section{F. Feature Extraction}

In this study, four popular EMG features namely mean absolute value (MAV), root mean square (RMS), maximum fractal length (MFL) and wavelet energy (E) are utilized.

Mean absolute value (MAV) is a popular feature in the classification of EMG signals. In short, MAV is an average of the summation of absolute value of EMG signals [5], [20]. Mathematically, MAV can be calculated as:

$M A V_{i}=\frac{1}{L} \sum_{n=1}^{L}\left|D_{i, n}\right|$

where $D_{i}$ is the coefficient at $i$ frequency band and $L$ is referred to the length of coefficient.

Root mean square (RMS) is one of the famous features that describes the information related to muscle force and activation [21], [22]. RMS can be expressed as:

$$
R M S_{i}=\sqrt{\frac{1}{L} \sum_{n=1}^{L} D_{i, n}^{2}}
$$

where $D_{i}$ is the coefficient at $i$ frequency band and $L$ is referred to the length of coefficient.

Maximum fractal length (MFL) measures the low-level muscle activation and it is modified from wavelength and RMS features [23]. In mathematics, MFL can be represented as follows:

$$
M F L_{i}=\log \left(\sqrt{\sum_{n=1}^{L-1}\left(D_{i, n+1}-D_{i, n}\right)^{2}}\right)
$$

where $D_{i}$ is the coefficient at $i$ frequency band and $L$ is referred to the length of coefficient.

Wavelet energy (E) of the coefficients in each sub-band represents the energy distribution of EMG signal, and it can be written as [13]:

$E_{i}=\frac{1}{L} \sum_{n=1}^{L}\left(D_{i, n}\right)^{2}$

where $L$ is the length of the coefficient and $D_{i}$ is the coefficient at $i$ frequency band.

\section{RESULTS AND DISCUSSIONS}

In this paper, 12 different mother wavelets in both DWT and WPT are investigated. Remarkably, DWT and WPT transformed the EMG signals into multiresolution coefficients. The features are then extracted from each coefficient to form the feature vector. It is worth noting that the number of extracting features is based on the wavelet decomposition level. DWT at fourth decomposition results in 8 coefficients, thus 384 features ( 4 features $\times 8$ coefficients $\times 12$ channels) are extracted. On the contrary, WPT at third decomposition level produces 14 coefficients. In total, 672 features ( 4 features $\times 14$ coefficients $\times 12$ channels) are extracted from each movement from each subject. Before performance evaluation, the features are normalized in the range between 0 and 1 in order to prevent numerical problem.

Two machine learning algorithms (classifier) namely linear discriminate analysis (LDA) and support vector machine (SVM) with radial basis function are used in performance evaluation. LDA and SVM are chosen due to their promising performances in previous works [24], [25]. For performance evaluation, Ten-fold cross-validation is applied. The data set is 
randomly divided into 10 equal parts. Each part is used for testing in succession, while the remaining part is used in training session. The classification results of ten folds are then averaged and recorded.

\section{A. Best Mother Wavelet in DWT}

Table III outlines the average class-wise accuracy (classification accuracy of 12 finger motions) of 12 mother wavelets of DWT across 10 subjects. Obviously, LDA showed better average class-wise performance as compared to SVM. Considering the optimal performance, the analysis of mother wavelet in LDA has served at prior. From the results, it can be inferred that the analysis of mother wavelet in DWT is significant important. As can be seen in Table III, each wavelet family offered different kind of classification performance. By using SVM, the best class-wise accuracy is achieved by Coif5 (88.8\%). Successively, Bior3.3 achieved the overall best mean class-wise accuracy, 95.23\%, followed by Bior2.2, 94.97\%. By contrast, the worst performance is obtained by Sym4, 92.99\%, followed by $\mathrm{Db} 6,93.82 \%$. In term of consistency, it is observed that Bior3.3 provided the most consistent and robust result due to smallest standard deviation (SD), $4.11 \%$. On the whole, Bior3.3 is found to be the most suitable mother wavelet function for the classification of 12 different finger motions. In short, Bior3.3 not only offers the best classification performance, but also provides highly consistent result. Hence, it can be concluded that DWT with Bior3.3 contributes the optimum performance in the classification of finger motions.

\section{B. Best Mother Wavelet in WPT}

The experimental results of WPT with 12 different mother wavelets are shown in Table IV. Based on the results obtained, it can be inferred that LDA outperformed SVM in this work. The possible reason might be the normalization of features, thus increasing the linearity of the feature model. From Table IV, each mother wavelet function provided different kind of responses in this work. This phenomenon indicates that the selection of mother wavelet is critically important. By applying SVM, the best mother wavelet in WPT is found to be Coif4, $87.04 \%$, followed by Sym6 and Sym8, 86.78\%. By employing LDA, all mother wavelets had their mean class-wise accuracy above $94 \%$. This implies that 12 finger motions have been classified very well. As can be seen in Table IV, Bior2.2 achieved the best recognition rate of $95.64 \%$ when LDA is utilized. Sym6 and Sym8 ranked the second best mother wavelets with mean class-wise accuracy of $95.63 \%$. On the other hand, Bior3.3 offered the most consistent result with the smallest standard deviation value, 3.68\%. Overall, it is observed that Bior2.2 not only provided the optimum results, but also afforded the consistent performance. Evidently, Bior2.2 is known to be the most suitable mother wavelet in WPT for the classification of finger motions.

TABLE III. AVERAGE Class-Wise ACCURACY OF 12 DifFERENT Mother WaVELETS OF DWT ACROSS 10 SubJeCtS

\begin{tabular}{|c|c|c|c|c|c|c|c|c|c|c|c|c|c|c|c|}
\hline \multirow{2}{*}{$\begin{array}{l}\text { Mother } \\
\text { wavelet }\end{array}$} & \multirow{2}{*}{$\begin{array}{l}\text { Types of } \\
\text { classifier }\end{array}$} & \multicolumn{14}{|c|}{ Class-wise accuracy (\%) } \\
\hline & & $F 1$ & $F 2$ & $F 3$ & F4 & $F 5$ & F6 & $F 7$ & $F 8$ & $F 9$ & $F 10$ & $F 11$ & $F 12$ & Mean & $S D$ \\
\hline \multirow{2}{*}{ Bior2.2 } & LDA & 94.05 & 95.48 & 95.71 & 98.33 & 98.57 & 98.57 & 98.33 & 93.39 & 84.76 & 98.33 & 88.65 & 95.48 & 94.97 & 4.35 \\
\hline & SVM & 83.05 & 88.71 & 90.65 & 93.71 & 92.05 & 89.76 & 87.90 & 91.55 & 78.52 & 85.95 & 76.52 & 93.21 & 87.63 & 5.63 \\
\hline \multirow{2}{*}{ Bior3.3 } & LDA & 95.48 & 94.29 & 97.14 & 98.57 & 98.57 & 97.14 & 100 & 95.71 & 86.33 & 93.57 & 88.48 & 97.50 & 95.23 & 4.11 \\
\hline & SVM & 82.55 & 89.31 & 89.23 & 95.48 & 92.05 & 85.83 & 87.57 & 93.21 & 77.56 & 85.95 & 77.52 & 92.14 & 87.37 & 5.82 \\
\hline \multirow{2}{*}{ Bior4.4 } & LDA & 96.90 & 94.40 & 94.29 & 98.33 & 98.57 & 100 & 98.33 & 95.48 & 80.12 & 95.24 & 89.58 & 95.71 & 94.75 & 5.35 \\
\hline & SVM & 84.95 & 91.81 & 90.65 & 93.71 & 94.90 & 88.69 & 88.24 & 92.98 & 79.71 & 85.95 & 76.52 & 93.21 & 88.45 & 5.77 \\
\hline \multirow{2}{*}{ Coif3 } & LDA & 97.14 & 93.57 & 97.14 & 98.33 & 97.50 & 100 & 96.90 & 95.48 & 84.75 & 97.14 & 83.93 & 95.71 & 94.80 & 5.13 \\
\hline & SVM & 83.29 & 89.81 & 90.65 & 93.71 & 94.90 & 89.76 & 88.14 & 92.98 & 79.05 & 85.95 & 75.33 & 93.21 & 88.07 & 6.13 \\
\hline \multirow{2}{*}{ Coif4 } & LDA & 90.86 & 93.21 & 98.57 & 100 & 96.90 & 98.57 & 98.57 & 93.15 & 81.93 & 95.48 & 84.62 & 95.71 & 93.97 & 5.70 \\
\hline & SVM & 84.71 & 90.38 & 92.08 & 93.71 & 94.90 & 88.69 & 89.67 & 89.29 & 78.46 & 86.95 & 77.52 & 92.14 & 88.21 & 5.53 \\
\hline \multirow{2}{*}{ Coif5 } & LDA & 95.48 & 95.48 & 93.21 & 100 & 94.64 & 98.33 & 97.14 & 94.29 & 81.55 & 98.57 & 86.95 & 97.14 & 94.40 & 5.25 \\
\hline & SVM & 83.52 & 91.81 & 90.65 & 93.71 & 94.90 & 89.76 & 88.48 & 92.98 & 82.25 & 85.95 & 78.38 & 93.21 & 88.80 & 5.22 \\
\hline \multirow{2}{*}{$\mathrm{Db} 4$} & LDA & 94.05 & 91.79 & 94.64 & 98.33 & 96.90 & 98.33 & 95.71 & 95.71 & 87.38 & 96.90 & 85.74 & 98.57 & 94.51 & 4.22 \\
\hline & SVM & 84.71 & 91.81 & 92.08 & 93.71 & 93.48 & 88.93 & 89.90 & 89.29 & 78.33 & 85.95 & 75.69 & 92.14 & 88.00 & 5.86 \\
\hline \multirow{2}{*}{ Db6 } & LDA & 95.14 & 90.95 & 93.71 & 98.33 & 96.07 & 97.14 & 96.90 & 94.05 & 82.54 & 97.14 & 86.73 & 97.14 & 93.82 & 4.82 \\
\hline & SVM & 86.14 & 90.38 & 92.08 & 93.71 & 94.90 & 87.26 & 90.67 & 90.71 & 79.79 & 86.95 & 77.52 & 92.64 & 88.57 & 5.37 \\
\hline \multirow{2}{*}{ Db8 } & LDA & 95.14 & 93.81 & 100 & 100 & 96.07 & 97.14 & 97.14 & 92.86 & 86.48 & 98.57 & 83.36 & 96.90 & 94.79 & 5.14 \\
\hline & SVM & 84.29 & 90.38 & 89.23 & 95.14 & 93.48 & 87.26 & 89.57 & 92.98 & 81.58 & 85.95 & 77.19 & 92.14 & 88.27 & 5.29 \\
\hline \multirow{2}{*}{ Sym4 } & LDA & 92.62 & 92.14 & 92.38 & 96.90 & 98.57 & 98.57 & 98.57 & 93.39 & 80.13 & 95.48 & 81.83 & 95.24 & 92.99 & 6.11 \\
\hline & SVM & 86.14 & 90.38 & 92.08 & 93.71 & 93.48 & 87.26 & 90.33 & 90.71 & 79.79 & 86.95 & 77.52 & 92.64 & 88.42 & 5.22 \\
\hline \multirow{2}{*}{ Sym6 } & LDA & 96.90 & 92.64 & 93.71 & 96.9 & 98.57 & 98.57 & 98.57 & 94.82 & 81.80 & 98.57 & 84.73 & 95.24 & 94.25 & 5.55 \\
\hline & SVM & 88.14 & 90.62 & 92.08 & 93.71 & 94.90 & 87.26 & 89.24 & 90.71 & 79.56 & 86.95 & 77.52 & 92.64 & 88.61 & 5.33 \\
\hline \multirow{2}{*}{ Sym8 } & LDA & 96.90 & 92.64 & 93.71 & 96.9 & 98.57 & 98.57 & 98.57 & 94.82 & 81.8 & 98.57 & 84.73 & 95.24 & 94.25 & 5.55 \\
\hline & SVM & 88.14 & 90.62 & 92.08 & 93.71 & 94.9 & 87.26 & 89.24 & 90.71 & 79.56 & 86.95 & 77.52 & 92.64 & 88.61 & 5.33 \\
\hline
\end{tabular}


TABLE IV. AVERAGE Class-Wise ACCURACY OF 12 DIFFERENT MOTHER WAVELETS OF WPT ACROSS 10 SUBJeCTS

\begin{tabular}{|c|c|c|c|c|c|c|c|c|c|c|c|c|c|c|c|}
\hline \multirow{2}{*}{$\begin{array}{l}\text { Mother } \\
\text { wavelet }\end{array}$} & \multirow{2}{*}{$\begin{array}{l}\text { Types of } \\
\text { classifier }\end{array}$} & \multicolumn{14}{|c|}{ Class-wise accuracy (\%) } \\
\hline & & $F 1$ & $F 2$ & $F 3$ & $F 4$ & $F 5$ & F6 & $F 7$ & $F 8$ & $F 9$ & $F 10$ & $F 11$ & $F 12$ & Average & $S D$ \\
\hline \multirow{2}{*}{ Bior2.2 } & LDA & 98.57 & 96.90 & 94.05 & 100 & 98.57 & 98.57 & 94.05 & 94.64 & 83.63 & 98.57 & 91.55 & 98.57 & 95.64 & 4.58 \\
\hline & SVM & 83.62 & 88.98 & 84.52 & 92.38 & 83.48 & 88.69 & 85.89 & 92.38 & 74.63 & 85.05 & 75.17 & 88.57 & 85.28 & 5.72 \\
\hline \multirow{2}{*}{ Bior3.3 } & LDA & 94.90 & 95.24 & 96.90 & 100 & 94.29 & 94.82 & 94.05 & 100 & 92.05 & 95.48 & 85.80 & 95.24 & 94.90 & 3.68 \\
\hline & SVM & 77.86 & 87.17 & 85.88 & 92.05 & 87.14 & 90.71 & 80.48 & 94.05 & 71.60 & 82.96 & 75.54 & 86.14 & 84.30 & 6.84 \\
\hline \multirow{2}{*}{ Bior4.4 } & LDA & 96.90 & 95.00 & 94.05 & 100 & 97.14 & 98.57 & 97.14 & 97.14 & 86.48 & 98.33 & 87.29 & 98.57 & 95.55 & 4.35 \\
\hline & SVM & 80.14 & 83.15 & 83.10 & 92.38 & 85.29 & 90.30 & 85.48 & 93.21 & 86.22 & 83.86 & 74.55 & 91.00 & 85.72 & 5.42 \\
\hline \multirow{2}{*}{ Coif3 } & LDA & 90.62 & 92.38 & 94.29 & 100 & 96.07 & 98.57 & 98.57 & 96.07 & 88.95 & 98.57 & 88.71 & 98.57 & 95.12 & 4.05 \\
\hline & SVM & 77.33 & 84.90 & 80.46 & 92.40 & 87.31 & 92.62 & 86.55 & 92.86 & 72.03 & 85.36 & 74.05 & 94.40 & 85.02 & 7.62 \\
\hline \multirow{2}{*}{ Coif4 } & LDA & 96.90 & 91.31 & 95.48 & 100 & 98.57 & 100 & 94.29 & 97.14 & 85.37 & 98.57 & 83.26 & 98.57 & 94.96 & 5.57 \\
\hline & SVM & 84.81 & 90.32 & 81.56 & 92.74 & 89.64 & 91.19 & 88.71 & 94.64 & 83.08 & 86.31 & 72.95 & 88.57 & 87.04 & 5.88 \\
\hline \multirow{2}{*}{ Coif5 } & LDA & 98.57 & 94.05 & 96.90 & 100 & 98.57 & 97.50 & 98.57 & 97.14 & 88.39 & 95.48 & 83.12 & 98.57 & 95.57 & 4.98 \\
\hline & SVM & 85.05 & 88.50 & 80.13 & 92.38 & 89.79 & 92.62 & 85.83 & 93.21 & 74.92 & 82.86 & 75.64 & 88.98 & 85.83 & 6.33 \\
\hline \multirow{2}{*}{$\mathrm{Db} 4$} & LDA & 92.86 & 91.90 & 94.64 & 100 & 96.07 & 100 & 96.90 & 98.33 & 90.83 & 98.57 & 85.71 & 98.57 & 95.37 & 4.34 \\
\hline & SVM & 83.81 & 89.21 & 83.32 & 92.05 & 88.81 & 90.12 & 89.07 & 89.29 & 76.22 & 82.80 & 72.13 & 86.83 & 85.31 & 6.01 \\
\hline \multirow[t]{2}{*}{ Db6 } & LDA & 94.90 & 88.81 & 94.40 & 100 & 96.07 & 98.57 & 100 & 97.14 & 89.19 & 97.14 & 84.93 & 98.57 & 94.98 & 4.86 \\
\hline & SVM & 81.07 & 91.15 & 82.23 & 92.40 & 89.31 & 91.19 & 86.50 & 93.21 & 84.06 & 84.88 & 74.04 & 89.57 & 86.63 & 5.66 \\
\hline \multirow[t]{2}{*}{ Db8 } & LDA & 96.90 & 93.81 & 94.40 & 100 & 98.57 & 98.33 & 94.29 & 95.71 & 89.23 & 98.57 & 85.26 & 100 & 95.42 & 4.46 \\
\hline & SVM & 85.64 & 88.65 & 82.46 & 93.81 & 92.40 & 89.23 & 83.83 & 93.21 & 73.03 & 83.69 & 77.11 & 86.67 & 85.81 & 6.33 \\
\hline \multirow{2}{*}{ Sym4 } & LDA & 93.71 & 94.05 & 97.14 & 98.00 & 98.57 & 98.57 & 98.57 & 96.07 & 92.62 & 98.33 & 83.12 & 98.33 & 95.59 & 4.48 \\
\hline & SVM & 81.95 & 88.48 & 79.89 & 92.74 & 89.64 & 88.93 & 92.33 & 94.64 & 77.46 & 85.95 & 75.68 & 91.07 & 86.56 & 6.35 \\
\hline \multirow{2}{*}{ Sym6 } & LDA & 92.98 & 95.24 & 97.14 & 100 & 97.14 & 98.57 & 97.14 & 97.50 & 92.62 & 96.90 & 83.95 & 98.33 & 95.63 & 4.26 \\
\hline & SVM & 84.36 & 87.58 & 79.56 & 92.74 & 91.31 & 90.12 & 89.48 & 94.64 & 82.56 & 84.76 & 74.64 & 89.57 & 86.78 & 5.81 \\
\hline \multirow{2}{*}{ Sym8 } & LDA & 92.98 & 95.24 & 97.14 & 100 & 97.14 & 98.57 & 97.14 & 97.50 & 92.62 & 96.90 & 83.95 & 98.33 & 95.63 & 4.26 \\
\hline & SVM & 84.36 & 87.58 & 79.56 & 92.74 & 91.31 & 90.12 & 89.48 & 94.64 & 82.56 & 84.76 & 74.64 & 89.57 & 86.78 & 5.81 \\
\hline
\end{tabular}

\section{CONCLUSION}

In this study, the best mother wavelet for DWT and WPT for EMG signals classification is presented. Ultimately, WT with the most suitable mother wavelet affirms the optimum classification performance, which strengthens the recognition rate for the classification of 12 different finger motions. The experimental results illustrated DWT with Bior3.3 was able to provide the highest class-wise accuracy in DB4. On the other side, Bior2.2 is found to be the most suitable mother wavelet in WPT due to its discriminate power in the classification of EMG signals. According to these findings, the best mother wavelet of DWT and WPP were coming from the Biorthogonal families. For such reason, we recommend that Biorthogonal families to be used in WT for the clinical and HMI applications. Future work will be focused on the selection of features in the process of mother wavelet selection, in which the best mother wavelet with the smallest number of features is guaranteed.

\section{ACKNOWLEDGMENT}

The authors would like to thank Skim Zamalah UTeM and Ministry of Higher Education (MOHE), Malaysia for funding research under grant GLuar/STEVIA/2016/FKECeRIA/100009.

\section{REFERENCES}

[1] N. Nazmi, M. A. Abdul Rahman, S.-I. Yamamoto, S. A. Ahmad, H. Zamzuri, and S. A. Mazlan, "A Review of Classification Techniques of EMG Signals during Isotonic and Isometric Contractions," Sensors, vol. 16, no. 8, p. 1304, Aug. 2016.

[2] M. N. M. Nor, R. Jailani, N. M. Tahir, I. M. Yassin, Z. I. Rizman, and R. Hidayat, "EMG Signals Analysis of BF and RF Muscles In Autism Spectrum Disorder (ASD) During Walking," Int. J. Adv. Sci. Eng. Inf. Technol., vol. 6, no. 5, pp. 793-798, Oct. 2016.

[3] R. N. Khushaba, A. Al-Timemy, S. Kodagoda, and K. Nazarpour, "Combined influence of forearm orientation and muscular contraction on EMG pattern recognition," Expert Syst. Appl., vol. 61, pp. 154-161, Nov. 2016.

[4] A. Subasi, "Classification of EMG signals using PSO optimized SVM for diagnosis of neuromuscular disorders," Comput. Biol. Med., vol. 43, no. 5, pp. 576-586, Jun. 2013. 
[5] A. Phinyomark, C. Limsakul, and P. Phukpattaranont, "Application of Wavelet Analysis in EMG Feature Extraction for Pattern Classification," Meas. Sci. Rev., vol. 11, no. 2, pp. 45-52, 2011.

[6] J. Too, A. R. Abdullah, N. M. Saad, N. M. Ali, and H. Musa, "A Detail Study of Wavelet Families for EMG Pattern Recognition," Int. J. Electr. Comput. Eng. IJECE, vol. 8, no. 6, pp. 4221-4229, Dec. 2018.

[7] F. A. Omari, J. Hui, C. Mei, and G. Liu, "Pattern Recognition of Eight Hand Motions Using Feature Extraction of Forearm EMG Signal," Proc. Natl. Acad. Sci. India Sect. Phys. Sci., vol. 84, no. 3, pp. 473-480, Sep. 2014.

[8] A. Phinyomark, A. Nuidod, P. Phukpattaranont, and C. Limsakul, "Feature Extraction and Reduction of Wavelet Transform Coefficients for EMG Pattern Classification," Elektron. Ir Elektrotechnika, vol. 122, no. 6, pp. 27-32, Jul. 2012.

[9] M. Hariharan, C. Y. Fook, R. Sindhu, B. Ilias, and S. Yaacob, “A comparative study of wavelet families for classification of wrist motions," Comput. Electr. Eng., vol. 38, no. 6, pp. 1798-1807, Nov. 2012.

[10] J. Too, A. R. Abdullah, N. Mohd Saad, and W. Tee, "EMG Feature Selection and Classification Using a Pbest-Guide Binary Particle Swarm Optimization," Computation, vol. 7, no. 1, p. 12, Mar. 2019.

[11] M. Atzori et al., "Effect of clinical parameters on the control of myoelectric robotic prosthetic hands.," J. Rehabil. Res. Dev., vol. 53, no. 3, pp. 345-358, 2016.

[12] S. Pizzolato, L. Tagliapietra, M. Cognolato, M. Reggiani, H. Müller, and M. Atzori, "Comparison of six electromyography acquisition setups on hand movement classification tasks," PLOS ONE, vol. 12, no. 10, p. e0186132, Oct. 2017.

[13] E. Gokgoz and A. Subasi, "Comparison of decision tree algorithms for EMG signal classification using DWT," Biomed. Signal Process. Control, vol. 18, pp. 138-144, Apr. 2015

[14] A. B. M. S. U. Doulah, S. A. Fattah, W. P. Zhu, and M. O. Ahmad, "Wavelet Domain Feature Extraction Scheme Based on Dominant Motor Unit Action Potential of EMG Signal for Neuromuscular Disease Classification,” IEEE Trans. Biomed. Circuits Syst., vol. 8, no. 2, pp. 155-164, Apr. 2014.

[15] R. H. Chowdhury, M. B. I. Reaz, M. A. B. M. Ali, A. A. A. Bakar, K. Chellappan, and T. G. Chang, "Surface electromyography signal processing and classification techniques," Sensors, vol. 13, no. 9, pp. 12431-12466, 2013.

[16] A. Subasi, "Classification of EMG signals using combined features and soft computing techniques," Appl. Soft Comput., vol. 12, no. 8, pp. 2188-2198, Aug. 2012.

[17] R. N. Khushaba, S. Kodagoda, S. Lal, and G. Dissanayake, "Driver Drowsiness Classification Using Fuzzy Wavelet-Packet-Based FeatureExtraction Algorithm," IEEE Trans. Biomed. Eng., vol. 58, no. 1, pp. 121-131, Jan. 2011.

[18] J. Rafiee, M. A. Rafiee, N. Prause, and M. P. Schoen, "Wavelet basis functions in biomedical signal processing," Expert Syst. Appl., vol. 38, no. 5, pp. 6190-6201, May 2011.

[19] N. M. Kakoty, A. Saikia, and S. M. Hazarika, "Exploring a family of wavelet transforms for EMG-based grasp recognition," Signal Image Video Process., vol. 9, no. 3, pp. 553-559, Mar. 2015.

[20] G. Purushothaman and R. Vikas, "Identification of a feature selection based pattern recognition scheme for finger movement recognition from multichannel EMG signals," Australas. Phys. Eng. Sci. Med., vol. 41, no. 2, pp. 549-559, Jun. 2018.

[21] K. S. Kim, H. H. Choi, C. S. Moon, and C. W. Mun, "Comparison of knearest neighbor, quadratic discriminant and linear discriminant analysis in classification of electromyogram signals based on the wrist-motion directions," Curr. Appl. Phys., vol. 11, no. 3, pp. 740-745, May 2011.

[22] O. S. Powar, K. Chemmangat, and S. Figarado, "A novel pre-processing procedure for enhanced feature extraction and characterization of electromyogram signals," Biomed. Signal Process. Control, vol. 42, pp. 277-286, Apr. 2018.

[23] A. Phinyomark, P. Phukpattaranont, and C. Limsakul, "Fractal analysis features for weak and single-channel upper-limb EMG signals," Expert Syst. Appl., vol. 39, no. 12, pp. 11156-11163, Sep. 2012.

[24] P. A. Karthick, D. M. Ghosh, and S. Ramakrishnan, "Surface electromyography based muscle fatigue detection using high-resolution time-frequency methods and machine learning algorithms," Comput. Methods Programs Biomed., vol. 154, no. Supplement C, pp. 45-56, Feb. 2018.

[25] X. Jiang, L.-K. Merhi, Z. G. Xiao, and C. Menon, "Exploration of Force Myography and surface Electromyography in hand gesture classification,” Med. Eng. Phys., vol. 41, pp. 63-73, Mar. 2017. 